\title{
The Reliability Optimization and Redundance Control for the Aeronautic Electronic System
}

\author{
Jiaxue Liu and Chunhong Chai \\ Aviation Maintenance NCO Academy of Air Force Engineering University, Xinyang,464000, henan, china \\ E-mail: ljx631@sina.com
}

Keywords: aeronautic electron, reliability, redundance control, multiple objective programming

\begin{abstract}
From aeronautic equipment to aeronautic weapons, all use a large number of electronic element as it's cell. The reliability of aeronautic equipment system is dependent on the reliability of aeronautic electron system to largeer extent. The reliability of aeronautic electron system affects the aeronautic equipment's work and effectiveness directly. In this paper, a multiple objective programming model is set up from the electronic element's redundance control and a method is given to solve this problem from the mathematical analysis.
\end{abstract}

\section{Introduction}

The reliability is one of the important indicators that we are considering in the demonstration analysis and research of the aviation equipment and aviation weapon. The reliability of aeronautic equipment system is dependent on the reliability of aeronautic electron system in largeer extent. The reliability of aeronautic electron system affect the aeronautic equipment's work and effectiveness directly. And the improvement of the electronic system's reliability depends on the improving of the electronic component's reliability. However because of the limitation of production process and materials, when the reliability of the electronic components can't be improved further, to further improve the reliability of system, only use redundancy of the electronic component to realize. Due to the introduction of redundant components will lead to the increase of the system's size, weight and cost inevitably, it affected the mobility, survival ability and combat effectiveness of the aviation equipment and aviation weapon. Therefore when using electronic component redundancy to improve system reliability, We must also consider the system's size, weight and cost factors in order to achieve the overall optimization .

Suppose $R_{b}$ is the reliability of a single electronic component (or subsystems), then the reliability of the parallel system that is composed by $n$ independent and identical components is

$$
R_{p}=1-\left(1-R_{b}\right)^{n}
$$

Then the number of the redundant components is

$$
n=\frac{\ln \left(1-R_{p}\right)}{\ln \left(1-R_{b}\right)}
$$

Suppose $C_{b}, V_{b}$ and $G_{b}$ is the cost, volume and weight of the electronic component unit respectively, then the redundant system's cost, volume and weight can be expressed respectively as

$$
\begin{gathered}
C_{s}=n \cdot C_{b}=\frac{\ln \left(1-R_{p}\right)}{\ln \left(1-R_{b}\right)} \cdot C_{b} \\
V_{s}=n \cdot V_{b}=\frac{\ln \left(1-R_{p}\right)}{\ln \left(1-R_{b}\right)} \cdot V_{b}
\end{gathered}
$$




$$
G_{s}=n \cdot G_{b}=\frac{\ln \left(1-R_{p}\right)}{\ln \left(1-R_{b}\right)} \cdot G_{b}
$$

Although this redundant method can improve the system's reliability, but because it backup all the basic components for the same numbers, so it increased cost, volume and weight of the system. In order to both meet the requirements of the system's reliability and reasonably reduce system's size, weight and cost, we establish the redundancy optimization model as following.

\section{Redundancy Optimization Model Is Established}

Suppose the unit is constituted of by $k$ different basic components in series. The reliability, cost, volume and weight of the first $i$ element are $R_{i}, C_{i}, V_{i}$ and $G_{i}$ respectively. Our goal is to make the redundant system's cost, volume and weight reache the minimum under the premise of reliability meet certain requirements. In order to meet the requirements of system reliability, we adopt the following redundancy method. The first $i$ subsystem is composed of $i$ kind of basic component that consists of $n_{i}$ independent elements in parallel. If the reliability $R_{s p}$ that system requirements is large (close to 1 ), then the system's failure rate $F_{s p}=1-R_{s p}$ close to zero. The redundant reliability of the first $i$ subsystem is

$$
R_{p_{i}}=1-\left(1-R_{i}\right)^{n_{i}}=1-F_{i}^{n_{i}} \quad(i=1,2, \cdots, k)
$$

In the formula, $F_{i}$ is each basic component's failure rate of the first $i$ subsystem, Therefore the reliability of redundant systems is

$$
R_{s p}=\prod_{i=1}^{k} R_{p_{i}}=\prod_{i=1}^{k}\left(1-F_{i}^{n_{i}}\right)
$$

After the redundant, the system's cost, volume and weight are respectively $C\left(n_{i}\right)=\sum_{i=1}^{k} n_{i} C_{i}, V\left(n_{i}\right)=\sum_{i=1}^{k} n_{i} V_{i} \quad$ and $G\left(n_{i}\right)=\sum_{i=1}^{k} n_{i} G_{i}$. So we establish redundancy control optimization model as following

$$
\text { MOP1: }\left\{\begin{array}{l}
\min f=\min f\left[C\left(n_{i}\right), V\left(n_{i}\right), G\left(n_{i}\right)\right] \\
\text { s.t. } \prod_{i=1}^{k}\left(1-F_{i}^{n_{i}}\right) \geq R_{s p} \\
n_{i} \in Z^{+} \quad(i=1,2, \cdots k)
\end{array}\right.
$$

Obviously, this is a multi-objective nonlinear integer programming problem.

\section{To Solve the Optimization Model}

The method of calculating the MOP1 optimization model is discussed below. Because nonlinear integer programming problem has no efficient algorithm up to now. So we find out MOP1 continuous optimal solution firstly, then use the enumeration method and multi-index decision method to find the satisfied integer solutions.

Because of the complexity of the constraint condition of MOP1, whether using the linear weighted, ideal point or target constraint method, turn it into a single objective planning to deal with is very complicated or impossible. Below we through the variable transformation, and combine mathematics analysis method and mathematical programming method, to solve the problem satisfactorily.

We make variable substitution :

$$
R_{p_{i}}=R_{s p}^{\beta_{i}} \quad\left(i=1,2, \cdots, k ; \beta_{i}>0\right)
$$


By (2), $R_{s p}=\prod_{i=1}^{k} R_{p_{i}}=\prod_{i=1}^{k} R_{s p}^{\beta_{i}}=R_{s p}^{\sum_{i=1}^{k} \beta_{i}}$.

Requirements the redundant system's reliability is not less than $R_{s p}$, it must be $\sum_{i=1}^{k} \beta_{i} \leq 1$. And because $R_{p_{i}}=1-F_{i}^{n_{i}}$, that is $R_{s p}^{\beta_{i}}=1-F_{i}^{n_{i}}$, thus

$$
n_{i}=\frac{\ln \left(1-R_{s p}^{\beta_{i}}\right)}{\ln F_{i}}
$$

The (3),(4) into MOP1, get the equivalent optimization model of MOP1:

MOP2: $\left\{\begin{array}{l}\min C=\min \sum_{i=1}^{k} \frac{C_{i}}{\ln F_{i}} \ln \left(1-R_{s p}^{\beta_{i}}\right) \\ \min V=\min \sum_{i=1}^{k} \frac{V_{i}}{\ln F_{i}} \ln \left(1-R_{s p}^{\beta_{i}}\right) \\ \min G=\min \sum_{i=1}^{k} \frac{G_{i}}{\ln F_{i}} \ln \left(1-R_{s p}^{\beta_{i}}\right) \\ \text { s.t. } \sum_{i=1}^{k} \beta_{i} \leq 1\end{array}\right.$

Theorem: MOP2 is a convex programming.

Prove: Easy to see, the feasible region $\sum_{i=1}^{k} \beta_{i} \leq 1$ is a convex domain. The hessian matrix $\nabla^{2} C$ of the cost function $C$ in the feasible is

$$
\left[\begin{array}{cccc}
\frac{-C_{1} R_{s p}^{\beta_{1}} \ln ^{2} R_{s p}}{\left(1-R_{s p}^{\beta_{1}}\right)^{2} \ln F_{1}} & & & \\
& \frac{-C_{2} R_{s p}^{\beta_{2}} \ln ^{2} R_{s p}}{\left(1-R_{s p}^{\beta_{2}}\right)^{2} \ln F_{2}} & & \\
& & \ddots & \\
& & \frac{-C_{k} R_{s p}^{\beta_{k}} \ln ^{2} R_{s p}}{\left(1-R_{s p}^{\beta_{k}}\right)^{2} \ln F_{k}}
\end{array}\right]
$$

Because $\ln F_{i}<0$, Easy to see the $\nabla^{2} C$ is a

positive definite matrix. Thus the first objective function $C$ is a convex function. In the same way, we can prove that the second and the third objective function is convex function. So the MOP2 is a convex programming. By the nonlinear programming theory, MOP2's local optimal solution is global optimal solution.

We use linear weighted method converted MOP2 into a single objective programming to solve. Through $\alpha$ - method or Delphi method method to geive the three target weight coefficient $w=\left(w_{1}, w_{2}, w_{3}\right)$, among them $w_{i}>0$, and $\sum_{i=1}^{3} w_{i}=1$. Converted MOP2 into its evaluation function optimization problem:

$$
\begin{aligned}
& p(w):\left\{\begin{array}{l}
\min H=\min \left(w_{1} C+w_{2} V+w_{3} G\right) \\
\text { s.t. } \sum_{i=1}^{k} \beta_{i} \leq 1
\end{array}\right. \\
& \text { Namely } p(w):\left\{\begin{array}{l}
\min \sum_{i=1}^{k} \frac{w_{1} C_{i}+w_{2} V_{i}+w_{3} G_{i}}{\ln F_{i}} \ln \left(1-R_{s p}^{\beta_{i}}\right) \\
\text { s.t. } \sum_{i=1}^{k} \beta_{i} \leq 1
\end{array}\right.
\end{aligned}
$$

By the nonlinear programming theory, the optimal solution of $p(w)$ is MOP2's non inferior 
solution. But it is very difficult to solve $p(w)$ because of the complexity of the objective function. We put its inequality constraints into equality constraint firstly. Then wo using the Lagrange multiplier method and equivalent infinitesimal substitution to solve the problem easily and obtain satisfactory results.

We construct the Lagrangian function

$$
\begin{aligned}
& L=\sum_{i=1}^{k} \frac{w_{1} C_{i}+w_{2} V_{i}+w_{3} G_{i}}{\ln F_{i}} \ln \left(1-R_{s p}^{\beta_{i}}\right)+\lambda\left(\sum_{i=1}^{k} \beta_{i}-1\right) \\
& \stackrel{\Delta}{=} \sum_{i=1}^{k} A_{i} \ln \left(1-R_{s p}^{\beta_{i}}\right)+\lambda\left(\sum_{i=1}^{k} \beta_{i}-1\right)
\end{aligned}
$$

Make $\frac{\partial L}{\partial \beta_{i}}=\frac{-A_{i} R_{s p}^{\beta_{i}}}{1-R_{s p}^{\beta_{i}}} \ln R_{s p}+\lambda=0$

$\frac{\partial L}{\partial \lambda}=\sum_{i=1}^{k} \beta_{i}-1=0$

Get $\sum_{i=1}^{k} \beta_{i}=1, \lambda=\frac{A_{i} R_{s p}^{\beta_{i}}}{1-R_{s p}^{\beta_{i}}} \ln R_{s p}$

By (3.3), get $\beta_{i}=\frac{1}{\ln R_{s p}} \ln \left(\frac{\lambda}{\lambda+A_{i} \ln R_{s p}}\right)$

Thus $\sum_{i=1}^{k} \beta_{i}=\frac{1}{\ln R_{s p}}\left[\sum_{i=1}^{k} \ln \left(\frac{\lambda}{\lambda+A_{i} \ln R_{s p}}\right)\right]$

$=\frac{-1}{\ln R_{s p}}\left[\sum_{i=1}^{k} \ln \left(1+\frac{A_{i} \ln R_{s p}}{\lambda}\right)\right]$

When the required reliability of the system is $R_{s p} \approx 1$,

By (5), $\frac{A_{i} \ln R_{s p}}{\lambda}=\frac{1-R_{s p}^{\beta_{i}}}{R_{s p}^{\beta_{i}}} \approx 0$.

And by the principle of equivalent infinitesimal substitution in mathematical analysis, when $x \approx 0$, there are $\ln (1+x) \approx x$. So to reduction (6)

$$
\begin{aligned}
& \sum_{i=1}^{k} \beta_{i}=\frac{-1}{\ln R_{s p}}\left[\sum_{i=1}^{k} \ln \left(1+\frac{A_{i} \ln R_{s p}}{\lambda}\right)\right] \\
& \approx \frac{-1}{\ln R_{s p}} \sum_{i=1}^{k} \frac{A_{i} \ln R_{s p}}{\lambda}=\frac{-1}{\lambda} \sum_{i=1}^{k} A_{i} \\
& \text { By } \sum_{i=1}^{k} \beta_{i}=1, \text { get } \lambda=-\sum_{i=1}^{k} A_{i}
\end{aligned}
$$

The (8) into (6), get

$$
\begin{aligned}
& \beta_{i}=\frac{1}{\ln R_{s p}} \ln \left[\frac{-\sum_{i=1}^{k} A_{i}}{-\sum_{i=1}^{k} A_{i}+A_{i} \ln R_{s p}}\right] \\
& =\frac{-1}{\ln R_{s p}} \ln \left[1-A_{i} \ln R_{s p} / \sum_{i=1}^{k} A_{i}\right]
\end{aligned}
$$




$$
\begin{aligned}
& =\frac{-1}{\ln R_{s p}} \ln \left[1+A_{i} \ln R_{s p} / \lambda\right] \\
& \approx \frac{-1}{\ln R_{s p}} \cdot \frac{A_{i} \ln R_{s p}}{\lambda} \\
& =\frac{-A_{i}}{\lambda}=A_{i} / \sum_{j=1}^{k} A_{j}
\end{aligned}
$$

So we get the approximate optimal solution of $p(w)$,that is MOP2's approximate pareto solutions.

$$
\begin{aligned}
& \beta_{i}=\frac{A_{i}}{\sum_{j=1}^{k} A_{j}} \\
& =\frac{\left(w_{1} C_{i}+w_{2} V_{i}+w_{3} G_{i}\right) / \ln F_{i}}{\sum_{j=1}^{k}\left[\left(w_{1} C_{j}+w_{2} V_{j}+w_{3} G_{j}\right) / \ln F_{j}\right]}
\end{aligned}
$$

By (9) calculate $\beta_{i}$. Plug in (4) calculate $n_{i}$ and enumerated integer we can find out optimal redundant numbers. Then plug in MOP1 we can get satisfactory solution of original problem. This kind of method provides a feasible algorithm for the reliability optimization and redundancy control problems.

\section{Case Analysis}

A system in a certain type fighter is composed of three elements. The cost, volume, weight and failure rate of each element are shown in table 1. Requirements through the redundant configuration to make the system's reliability is $R_{s p}=0.98$ and to make the cost, volume and weight as small as possible. The three targets weights of cost, volume and weight is $w=(0.25,0.45,0.30)$ after investigation and analysis. How should we redundancy and configure each element's number can meet our requirements and satisfaction goal.

Analyse: By (3.7), (3.2), we get $\beta_{i}$ and $n_{i}$ in the last two columns of table 1 . In the continuous solutions of $n_{i}$ enumerated integer. When $n_{2}=2$, then $R_{2}=0.975$ and $R_{s p}<0.97$, It did not meet the requirement of system reliability. Therefore, $n=2$ solutions are not feasible. Other redundancy schemes and the targets can be seen in table 2.

Among the four schemes in table 2, scheme 2 can not meet the needs of MOP1 constraints, so it is not feasible solution. The system reliability of scheme 1, 3, 4 were greater than 0.98 and meet the constraint conditions of MOP1, so they are feasible schemes.

In the feasible schemes, by three targets cost, volume and weight we can see, the three target values of redundancy scheme 1 are greater than (inferior to) the corresponding target values of scheme 3,4 . So the scheme 1 is a bad scheme. The three target values of scheme 3 are less than (better than) the corresponding target values of scheme 1, 4. So the scheme 3 is the best redundancy optimization scheme.

Table 1. The index values of the system components

\begin{tabular}{|c|c|c|c|c|c|c|}
\hline $\begin{array}{c}\text { element } \\
\text { number } \mathrm{i}\end{array}$ & $\begin{array}{c}\text { cost } \\
\mathrm{C}_{\mathrm{i}} \text { (million) }\end{array}$ & $\begin{array}{c}\text { volum } \\
\mathrm{V}_{\mathrm{i}}\left(\mathrm{cm}^{3}\right)\end{array}$ & $\begin{array}{c}\text { weight } \\
\mathrm{G}_{\mathrm{i}}(\mathrm{kg})\end{array}$ & $\begin{array}{c}\text { failure rate } \\
\mathrm{F}_{\mathrm{i}}\end{array}$ & $\beta_{i}$ & $n_{i}$ \\
\hline 1 & 1.1 & 5.5 & 1.5 & 0.12 & 0.4286 & 2.24 \\
\hline 2 & 0.8 & 4.6 & 0.8 & 0.15 & 0.3757 & 2.57 \\
\hline 3 & 0.6 & 3.2 & 0.5 & 0.08 & 0.1956 & 2.19 \\
\hline
\end{tabular}


Table 2. The scheme table of the redundant configuration

\begin{tabular}{|c|c|c|c|}
\hline redundancy schemes & system reliability & cost $\mathrm{C}_{\mathrm{i}}$ (million) & volum \\
\hline $\mathrm{V}_{\mathrm{i}}\left(\mathrm{cm}^{3}\right) \quad$ weight $\mathrm{G}_{\mathrm{i}}(\mathrm{kg})$ & & & \\
\hline scheme $1\left(n_{1}=n_{2}=n_{3}=3\right)$ & 0.994 & 7.5 & 39.9 \\
\hline 8.4 & & & \\
\hline scheme $2\left(n_{1}=n_{3}=2, n_{2}=3\right)$ & 0.976 & 5.6 & 29.8 \\
\hline 6.1 & & & \\
\hline $\begin{array}{l}\text { scheme } 3\left(n_{1}=2, n_{2}=n_{3}=3\right) \\
6.9\end{array}$ & 0.992 & 6.4 & 34.4 \\
\hline $\begin{array}{l}\text { scheme } 4\left(n_{1}=n_{2}=3, n_{3}=2\right) \\
7.9\end{array}$ & 0.991 & 6.9 & 36.7 \\
\hline
\end{tabular}

\section{Conclusion}

The system component's redundancy optimization configuration method that wo discussed is based on the strict mathematical programming theory and scientific analysis method. This method not only scientific and reliable, and convenient and feasible for the aircraft subsystem's reliability redundancy optimization.

\section{References}

[1] Xuan Jiaji, Multiple Objective Decision Making, Hunan Science and Technology Press, Changsha, China, 1989.

[2] Fredericks. Hiller, GeraldJ. liebeman, Introduction to Operations Research, Mc Graw-Hill Book Company, New York,1995.

[3] Xu Jiuping, Li Jun, The Theory and Method of Multiple Objective Decision Making, Tsinghua University Press, Beijing, China, 2005.

[4] Cheng Wuyi, The System Reliability Theory and Its Application, The University of Beijing Aeronautics and Astronautics Press Beijing, China, 2012.

[5] Shen Zhen, The measures to improve the reliability of electronic equipment system, Technology Trend, No.2, 247-249, 2011. 